\title{
The nine-dot problem: Beyond perceptual organization
}

\author{
CLARKE A. BURNHAM and KENNETH G. \\ DAVIS, University of Texas, Austin, Tex. \\ 78712
}

$A$ dozen versions of the nine-dot problem were individually administered. Instructions stating that $S$ could extend his lines beyond the boundary of the square formed by the nine dots were manginally effective in increasing the number of Ss who solved the problem in a 10 min period. Presenting two extra dots outside the square dramatically increased the number of solvers. It was concluded that perceptual organization, seeing the dots as a confining square, is a minor factor in making the problem a difficult one. The source of the difficulty is, instead, the fact that the sequence of lines involved in the solution is nonobvious.

The nine-dot problem (Fig. 1) is frequently used in introductory psychology courses and texts to illustrate the detrimental effects of set on problem solving. To solve the problem, a line must be drawn beyond the boundary of the square formed by the dots. The typical, and intuitively reasonable, explanation of the difficulty of the problem is that $S$ organizes the dots as a bounded square and therefore confines his solution attempts to the interior of the square (e.g., Kendler, 1968; Krech, Crutchfield, \& Livson, 1969).

In this investigation, we studied a dozen versions of the nine-dot problem to determine the effect of various instructions and modes of presentation on the time it took Ss to solve the problem. One instructional variable was to tell $S s$ that they could go outside the square formed by the dots. The presentation variable was to alter the square appearance of the problem by adding two dots outside the square formed by the nine dots. It was expected that these variables would remove the set created by the square arrangement of the dots and thus facilitate solving the problem. Other instructional variables were derived from an analysis of the solution attempts of students in an upper-division psychology of thought course, and from a discussion with them of the variables that they felt inhibited achieving the solution. These students reported that they tended to avoid drawing lines which crossed one another and avoided touching the same dot with more than one line. Furthermore, students who solved the problem tended to begin their solutions with the lower left dot, whereas the nonsolvers showed no preference for a starting point instructions used in the Series 1 problems were used in Series 2, with the addition of the sentence, "Start with the dot enclosed by the square."

Series 3 (11 dots). Two additional dots were presented in the Series 3 versions, as shown in Fig. 2. Two sets of instructions were used. These were: $3 A$ (no additional instructions) and $3 \mathrm{~B}$ (crossing and touching permitted).

Series 4 ( 11 dots and start at lower left). The two Series 4 versions contained 11 dots, as shown in Fig. 2, and the lower left dot was enclosed by a small square. The same two sets of instructions used in the Series 3 problems were used, with the addition of the instructions to start with the lowar left dot.

The nine-dot problem was given

Fig. 1. The nine-dot problem. Connect the dots by drawing four straight lines without retracing and without removing your pencil from the paper.

and often began with a dot from which the problem could not be solved. These variables were manipulated to evaluate their contribution to the difficulty of the problem.

\section{METHOD}

The nine-dot problem was mimeographed on $81 / 2 \times 11$ in. sheets. The dots were 2 in. apart, and the problem was centered on the sheet. The instructions, which were presented at the top of the sheet, started with the sentence, "Connect the dots by drawing four straight lines without retracing and without removing your pencil from the paper." They concluded with the sentence, "Continue working on the problem, erasing your pre-solution attempts, until you get the solution or until the experimenter stops you at the end of 10 minutes." Additional instructions, corresponding to the experimental treatments, were included between these sentences.

Twelve versions of the problem were used. These may be divided into four series:

Series 1 (standard form). The problem was presented as shown in Fig. 1. Four different sets of instructions were used. These were: $1 \mathrm{~A}$ (no additional instructions); $1 B$ (outside permitted), "The lines can extend beyond the square formed by the dots"; 1C (crossing and touching permitted), "The lines can cross one another and can touch the same dot more than once"; and 1D (outside and crossing and touching permitted), "The lines can extend beyond the square formed by the dots, and they can cross one another and can touch the same dot more than once."

Series 2 (start at lower left). The problem was presented as shown in Fig. 1, with the addition of a small square placed around the lower left dot. The same four sets of individually to $194 \mathrm{Ss}$ following their participation in various visual-perception experiments which were not concerned with set or perceptual organization. Five different Es administered the problem and recorded the solution times; they each ran an approximately equal proportion of $\mathrm{Ss}$ in the 12 versions. The Ss were University of Texas students fulfilling a portion of the introductory psychology course requirement.

After solving the problem, or working on it for $10 \mathrm{~min}$, each $\mathrm{S}$ answered the following questions: (1) Have you seen this or a similar problem before? (2) Have you seen the solution to this or a similar problem before? and (3) Did you recall the solution and use it for this problem in any way? The data from those 14 Ss who answered "yes" to Question 3 and who solved the problem were not included in the analysis. The experiment was continued until there were 15 usable Ss for each of the 12 versions of the problem.

\section{RESULTS}

The number of Ss, out of 15 , who solved their version of the problem in the allotted $10 \mathrm{~min}$, the median solution time, and the

\section{$\bullet$}

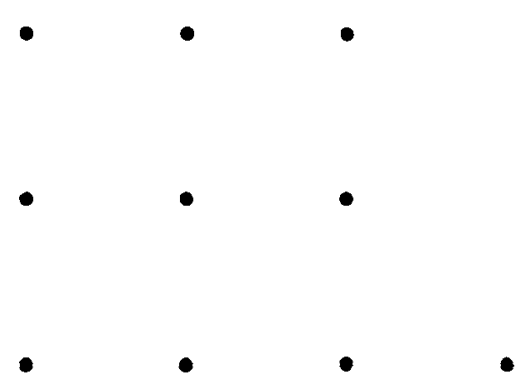

Fig. 2. The "nine-dot" problem as presented in Series 3 and 4. 
Table 1

Number of Solvers (Within $10 \mathrm{Min}$ ), Median Solution Times (Min), and Mean Solution Times (Min) for the 12 Versions of the Nine-Dot Problem

\begin{tabular}{|c|c|c|c|c|}
\hline & Version and Description & $\begin{array}{c}\text { Number } \\
\text { of } \\
\text { Solvers }\end{array}$ & $\begin{array}{c}\text { Median } \\
\text { Solution } \\
\text { Time } \\
\end{array}$ & $\begin{array}{c}\text { Mean } \\
\text { Solution } \\
\text { Time }\end{array}$ \\
\hline Series 1 & Standard form & & & \\
\hline $1 \mathrm{~A}$ & No additional instructions & 0 & 10.00 & 10.00 \\
\hline 1B & Outside permitted & 4 & 10.00 & 8.90 \\
\hline $1 \mathrm{C}$ & Crossing and touching permitted & 1 & 10.00 & 9.51 \\
\hline $1 \mathrm{D}$ & Outside and Crossing and touching permitted & 3 & 10.00 & 9.36 \\
\hline Series 2 & Start at lower left & & & \\
\hline $2 \mathrm{~A}$ & No additional instructions & 1 & 10.00 & 9.52 \\
\hline $2 \mathrm{~B}$ & Outside permitted & 3 & 10.00 & 9.01 \\
\hline $2 C$ & Crossing and touching permitted & 2 & 10.00 & 9.14 \\
\hline 2D & Outside and Crossing and touching permitted & 2 & 10.00 & 9.30 \\
\hline Series 3 & Eleven dots & & & \\
\hline $3 \mathbf{A}$ & No additional instructions & 12 & 4.40 & 5.44 \\
\hline 3B & Crossing and touching permitted & 12 & 4.35 & 5.17 \\
\hline Series 4 & Eleven dots and Start at lower left & & & \\
\hline $4 \mathrm{~A}$ & No additional instructions & 14 & 1.97 & 3.41 \\
\hline 4B & Crossing and touching permitted & 14 & 2.50 & 2.92 \\
\hline
\end{tabular}

mean solution time are presented in Table 1. Because of the skewed distribution of solution times for the Series 1 and 2 versions, the $\chi^{2}$ and Fisher exact-probability tests were used for statistical comparisons. 1

The instructions stating that the lines could extend beyond the square formed by the dots facilitated performance, although the increase in the number of solvers was of marginal statistical significance. The proportion of solvers among the Ss who received the outside-permitted instructions, Versions 1B and ID (23.3\%), approaches being significantly greater $(p=.052)$ than the proportion of solvers among the Ss who received Versions $1 \mathrm{~A}$ and $1 \mathrm{C}$ (3.3\%). However, comparable results were not obtained for the Series 2 versions. The proportion of solvers among the $S$ s who received Versions $2 \mathrm{~B}$ and $2 \mathrm{D}(16.7 \%)$ does not differ significantly $(p>.50)$ from the proportion of solvers among the Ss who received Versions $2 \mathrm{~A}$ and $2 \mathrm{C}(10.0 \%)$. If Series 1 and 2 are combined, the proportion of solvers among the Ss receiving the outside-permitted instructions (20.0\%) approaches being significantly greater $\left(x^{2}=3.53, \mathrm{df}=1, \mathrm{p}<.10\right)$ than the proportion of solvers among those Ss who did not receive these instructions (6.7\%).

The addition of the two extra dots clearly facilitated solving the problem. The proportion of solvers among those Ss who received the Series 3 and 4 versions of the problem $(86.7 \%)$ is significantly greater $\left(\chi^{2}=88.42\right.$, df $\left.=1, \mathrm{p}<.001\right)$ than the proportion of solvers among those Ss who recejved the Series 1 and 2 versions (13.3\%). The extra dots also resulted in significantly more solvers than the instructions stating that the lines could extend beyond the square formed by the dots $\left(\chi^{2}=50.93\right.$, $\mathrm{df}=1, \mathrm{p}<.001)$.

The instructions stating that the lines could cross one another and that they could touch the same dot more than once did not facilitate solving the problem. These instructions produced absolutely no increase in the proportion of solvers in any of the four series.

The instructions telling $S$ to start at the lower left dot failed to improve performance in the nine-dot versions of the problem. The number of solvers among the Series 2 Ss, who were given these starting instructions, is identical to the number of solvers among the Series 1 Ss. However, these instructions did increase the speed of solution for those $S s$ who received the 11-dot problem. The median solution time for the Ss in Series 3 , who were not told where to start, was $4.38 \mathrm{~min}$; the median solution time for the Ss in Series 4, who were told to start at the lower left, was $2.14 \mathrm{~min}$. The distribution of solution times of these two groups of Ss is significantly different (Mann-Whitney $U$ test, $z=2.20, p<.05$ ).

\section{DISCUSSION}

The fact that there were no solvers among the Ss who received the Series $1 \mathrm{~A}$ version attests to the difficulty of the nine-dot problem for college freshmen and sophomores. Special instructions or an alteration in the mode of presentation of the standard problem are apparently necessary to enable these Ss to solve the problem within a $10 \mathrm{~min}$ period. Contrary to our expectations based on the statements of upper-class students, instructions which explicitly permitted crossing lines and touching the same dot more than once did not facilitate solving the problem. Telling Ss to begin at a particular starting point likewise does not appear to aid in achieving the solution of the standard version of the nine-dot problem.

The typical interpretation of the difficulty of the nine-dot problem is that the square arrangement of the dots produces a self-imposed restraint on the attempted solutions to the problem. This perceptual organization interpretation is partially correct; Ss who are not told that they can go outside the square formed by the nine dots tend not to do so. Yet when this restraint is removed by verbal instructions, the percentage of Ss solving the problem does not change dramatically, even though the solution attempts of the Ss given these instructions do extend beyond the square formed by the dots. The hypothesis that the difficulty of the problem is due to a self-imposed perceptual organization does not account for these experimental data. The verbal instructions may or may not alter the perceptual organization. Such instructions do cause Ss to extend their lines beyond the boundary of the square. Doing so does not usually result in solving the problem.

Altering the form of the problem by presenting a particular arrangement of 11 dots does dramatically increase the number of solutions. It is unlikely that this is due to a removal of the perceptual organization of a square pattern, since removal of the restraint against going outside the boundary of the square is not, in itself, sufficient to produce a large increase in the number of solvers. It is probable that the presentation of 11 dots provided a direction and a rationale for drawing the necessary four lines for the solution. If $S$ starts with the lower left dot, a horizontal line to the right connects four dots. Upon reaching the far right dot, the dot outside the square, the diagonal line to the upper left dot, a dot outside the square, then maximizes the number of dots connected. The vertical line to the lower left is then the only reasonable line to draw and, finally, a diagonal line to the upper right dot solves the problem. This interpretation is supported by the finding that solution times were shortened when a reasonable starting point was indicated for the 11-dot problem. It should be noted that other positions of the two extra dots might misdirect $S$ and thus make the problem more difficult. In sum, it is our hypothesis that the ex tra dots presented in Series 3 and 4 facilitated achieving the solution because they provided clues for drawing the lines necessary to solve the problem. The nine-dot problem remains difficult to solve even when the restraints caused by perceptual organization are removed. ${ }^{2}$

\section{REFERENCES}

KENDLER, H. H, Basic psychology. (2nd ed.) New York: Appleton-Century-Crofts, 1968

KRECH, D., CRUTCHFIELD, R. S., \& LIVSON, N. Elements of psychology. New York: Knopf, 1969.

\section{NOTES}

1. All reported $p$ values are two-tailed. $\chi^{2}$ has been corrected for continuity.

2. Discussions with colleagues who have used 
this problem in class support this interpretation. They report that it is sometimes embarrassingly difficult to draw the solution, even through they know the principle involved. Similarly, the Es who administered the problem to the Ss in this study reported that it took them some time before they could rapidly draw the solution when demonstrating it to Ss.

\section{Rigidity as a function of task complexity}

RONALD RAY SCHMECK, Southern Illinois University, Carbondale, Ill. 62901, and FRED RIBICH, The Johns Hopkins Hospital, Baltimore, Md. 21205

Four groups of Ss were tested on hidden-word tasks consisting of 12 set-inducing items followed by 13 test items. One group, the simple-task group, received 7-letter items having a 4-letter word embedded in each. The other group, the complex-task group, received 10-letter items with each again having a 4-letter word embedded in it. The "set" response consisted of choosing alternate letters that formed a word, while the direct solution consisted of simply choosing 4 consecutive letters that formed a word. Direct solutions were possible only on the 13 test items. It was found that the complex experimental group produced significantly more direct solutions ("broke" the set more often) than did the simple experimental group. Results supported a behavioral model of rigidity based on the principles of Hull-Spence behavior theory.

To the present time, the literature contains no studies designed to investigate the interaction of task complexity and behavioral rigidity or mental set (see Luchins, 1959). Within the Hull-Spence system (Spence, 1956), a simple task is assumed to be one that involves few competing responses, with each of the competing responses having habit strengths that are significantly less than the habit strength of the dominant correct response. On the other hand, a complex task would be one having many competing responses with habit strengths that are almost equal. If it is assumed that behavioral variability is a function of the number and strengths of the competing responses possible within a particular situation, then it can be predicted that an $S$ will be more likely to "break" an established mental set while performing a complex task than while performing a simple task. The following study is designed to test this prediction using hidden-word tasks of varying complexity.

\section{SUBJECTS AND PROCEDURE}

The Ss were college freshmen enrolled in beginning psychology courses at Ohio University. They were tested in two groups of 30 each. One group received a simple list of hidden-word items, the other received a complex list. The simple list contained 25 items, each consisting of 7 letters. Ss were instructed to find a hidden 4-letter word without transposing the order of the letters. The first 12 items ("set-inducing" iter(s) could be solved, by choosing every other letter, beginning with the first letter. The last 13 items ("test" items) were solvable not only by the set method, but also by the more direct method of detecting a word composed of 4 consecutive letters embedded in the anagram. The complex list also contained 25 items, but in this case, each item was composed of 10 letters. Ss were given the same instructions as those given in the simple list. The first 12 items, again the set-inducing items, were solvable by the same alternating method as that used in the simple list, but the 1st letter of the alternating sequence varied from the first to the fourth position in the group of 10 letters. The remaining 13 items were again solvable both by the set method and by a more direct method like that of the simple list. The items were typed in capital letters, with 5 items to a page. Ss indicated their choice of letters by filling in a circle below each letter. Ss were also told not to go on to other items unless the previous item had been completed.

Two groups of five Ss each served as control groups. One group was given only the 13 test items from the simple list, while the other group was tested on only the 13 test items from the complex list. They were given the same instructions as the experimental groups.

\section{RESULTS AND DISCUSSION}

Several Ss in each of the two experimental groups failed to utilize the set method in solving the first 12 problems. Some produced nonsense words, and others skipped items. Thus, to insure that a set had indeed been formed, the following criteria were used to select those tests to be scored: (1) At least the eight items (Nos. 5-12) immediately preceding the test items were all solved by the set method, or (2) no more than three items were solved by a nonset method with none of these items being the last two of the set-inducing items (No. 11 and No. 12). Fourteen Ss in each of the two experimental groups met the criteria.

The control group produced $100 \%$ direct solutions of the 13 test items. The difference between the means of the complex experimental group (9.8) and the complex control group (13.0) was significant $[t(17)=3.68, p<.01]$. Also, the difference between the means of the simple experimental group (5.3) and the simple control group (13.0) was significant $[\mathrm{t}(17)=8.85, \mathrm{p}<.001]$. These significant differences indicate that the set-inducing items did, indeed, produce a set in both of the experimental groups.

The difference between the means of the simple and complex experimental grcups was highly significant $[\mathrm{t}(26)=3.60$, $\mathrm{p}<.01]$, with the complex group demonstrating more direct (nonset) solutions to the test items. Simply by varying the beginning position of the altemating sequence and by increasing the number of letters in the items, it was possible to produce a significant increase in set-breaking behavior. This increase is attributable to the increased behavioral variability resulting from the presence of more and stronger competing responses on the complex task.

REFERENCES

LUCHINS, A. S. Rigidity of behavior. Eugene: University of Oregon Books, 1959.

SPENCE, K. W. Behavior theory and conditioning. New Haven: Yale University Press, 1956. 